\title{
DEMOGRAPHICS AND CORRELATION OF RISK FACTORS FOR HEPATOCELLULAR CARCINOMA IN PATIENTS PRESENTING TO A TERTIARY CARE FACILITY IN PAKISTAN
}

\author{
Hafsa Rashid, Laima Alam, Muhammad Asif Farooq, Muhammad Zafar Ali \\ Pak Emirates Military Hospital/National University of Medical Sciences (NUMS) Rawalpindi Pakistan
}

\begin{abstract}
Objective: Demographics of HCC in Pakistan. Correlation of HCC with its possible etiology. Correlation of tumor aggressiveness with PCR status and anti-viral treatment.

Study Design: Cross sectional study.

Place and Duration of Study: Pak Emirates Military Hospital, Rawalpindi from Jul 2017 to Jun 2018.

Methodology: Patients with age $>18$ years presenting with space occupying lesion(s) of liver were confirmed to be HCC according to standard guidelines. The variables such as age, gender, presence of cirrhosis, etiology of cirrhosis, tumor staging, viral status through PCR study and the treatment offered were documented. Baseline descriptive data was reported as mean with SD for continuous variables. Chi square test was used to compare qualitative data.

Results: A total of 195 patients were enrolled for one year. Male population with HCC was in predominance (75.9\%). Sixty one percent of the afflicted population was having liver cirrhosis, 34.9\% had decompensated cirrhosis and $3.8 \%$ had no cirrhosis. HCV accounted for the bulk of patients with cirrhosis (82\%) followed by HBV (9.2\%), HBV and HCV co-infection (3.1\%), NASH and cryptogenic cirrhosis (1.5\% each). Majority got diagnosed with triphasic CECT scan Abdomen, only $3.6 \%$ needed liver biopsy for diagnosis. Majority $(43.6 \%)$ belonged to BCLC B. Viral PCR was positive for $58.5 \%$ and $73.8 \%$ of the patients were treatment naïve.

Conclusion: HCC shows highest rates seen in male patients presenting in old age. Gender, classes of cirrhosis, number of lesions, portal vein thrombosis and extrahepatic metastasis correlated with possible risk factors of HCC. Tumor aggressiveness correlated with PCR status and anti-viral treatment.
\end{abstract}

Keywords: HBV, HCC, HCV.

This is an Open Access article distributed under the terms of the Creative Commons Attribution License (http://creativecommons.org/licenses/by/4.0), which permits unrestricted use, distribution, and reproduction in any medium, provided the original work is properly cited.

\section{INTRODUCTION}

HCC is leading cause of death in the present world however it has a higher prevalence in South East Asia. Hepatocellular carcinoma is the most common primary tumor of the liver. It is seventh most common cancer over all the world and third most common cause of cancer related deaths in the world 1 . Etiology has taken a turn in recent years in Asia and Pakistan has been reported to have shown increased prevalence of $\mathrm{HCV}^{2}$. Its most common risk factors include Hepatitis viruses namely B and C, alcohol and non-alcoholic fatty liver disease. Rising incidence in NASH and NAFLD chronic liver diseases is also contributing to changing epidemiology in developing countries ${ }^{3}$.

HBV infection is the major cause of HCC in india. Most patients at the time of diagnosis report with underlying cirrhosis hence HCC is more common in cirrhotic livers ${ }^{4}$. Like Pakistan Indian male population is afflicted more with $\mathrm{HCC}^{5}$.

This can be intrahepatic metastasis or multi-

Correspondence: Dr Hafsa Rashid, Gastroenterology Department, Pak Emirates Military Hospital, Rawalpindi Pakistan

Received: 18 Apr 2019; revised received: 07 Jan 2020; accepted: 09 Jan 2020 centric development of carcinogenesis. However the former shows worse prognosis ${ }^{6}$.

Positive PCR status with no history of antiviral treatment are more likely to develop HCC as no treatment can lead speedily to cirrhosis. It is detectable mostly by imaging technique like CT Scan or MRI abdomen. CECT scan is the preferred modality.

In this article we try to highlight that despite development of direct acting anti virals there is still rise in HCC secondary to hepatitis $C$ virus. Tumor aggressiveness in terms of BCLC staging, number of lesions and portal vein thrombosis correlated with PCR status and anti-viral treatment and demographics of HCC in Pakistani population were looked into.

\section{METHODOLOGY}

This was cross sectional study and included all cases with a diagnosis of HCC who presented to Pak Emirates Military Hospital Rawalpindi aged 18-70 years, between July 2017 June 2018. Pat-ents were thoroughly examined after proper history taking and necessary investigations were sent. A sample of 195 patients was enrolled using $\mathrm{WHO}$ calculator with a reference prevalence of $15 \%$ according to study in Agha Khan University Pakistan ${ }^{7}$. Based on ASSLD 
guidelines (2017) the diagnosis was based upon triphasic contrast enhanced CT scan of abdomen, MRI abdomen and liver biopsy were done as per requirement. All patients with either $\mathrm{HBV}, \mathrm{HCV}$, co-infection and NAFLD as underlying etiologies were included in the study. Some were of underlying cryptogenic cirrhosis. These were diagnosed by doing liver biopsy. The study was approved by our hospital ethics committee.

The Data collected included demographics, Viral PCR status and alpha feto protein, anti-viral treatment, causes of chronic liver disease and documentation of liver cirrhosis. Liver cirrhosis was established by radiological characteristics and laboratory tests of hepatic synthetic function. The severity of cirrhosis was assessed through child turcotte pugh score.We recorded HCC characteristics, tumor number, tumor size, metastasis and in case of multiple lesions, the largest one was selected.

Portal vein tumor thrombosis, extrahepatic
ECOG functional status was also recorded. Eastern Cooperative Oncology Group (ECOG) is widely used by clinicians to assess the functional status in patients with various cancers.

Statistical Analysis

Data management and statistical analysis were performed with SPSS-19. Baseline descriptive data was reported as mean with standard deviation (SD) for continuous variables and frequencies and percen-tages for categorical variables. Fisher exact test was used to compare qualitative data. All $p$-values $\leq 0.05$ were considered statistically significant.

\section{RESULTS}

A total of 195 patients were enrolled for a total period of one year. The male population was in predominance, 148 among $195(79.5 \%)$ as compared to female population i.e. 47 (24.1\%) and showed an association with etiology by having $p$-value $<0.001$. The mean \pm SD age for the patients was $59 \pm 8.9$ with a mean \pm SD BMI of $21.5 \pm 2.8$ (table-I).

Table-I: Characteristics of patients with HCC in relation to the cause of cirrhosis.

\begin{tabular}{|c|c|c|c|c|c|c|c|c|}
\hline \multirow[b]{2}{*}{ Variable } & \multicolumn{8}{|c|}{ Frequency in $n(\%)$ or Mean \pm SD } \\
\hline & Total & $\mathrm{HCV}$ & HBV & $\mathrm{HCV} / \mathrm{HBV}$ & NASH & Cryptogenic & No cirrhosis & $p$-value \\
\hline No. of Patients & 195 & $160(82.1)$ & $18(9.2)$ & $6(3.1)$ & $3(1.5)$ & $3(1.5)$ & $5(2.6)$ & - \\
\hline Age (Years) & $59.8 \pm 8.9$ & $59.4 \pm 8.7$ & $61 \pm 10$ & $57.5 \pm 6.4$ & $61.7 \pm 15.6$ & $75 \pm 8$ & $59 \pm 10$ & - \\
\hline \multicolumn{9}{|l|}{ Gender } \\
\hline Male & $148(75.9)$ & $119(74.4)$ & $16(88.9)$ & $5(83.3)$ & $1(33.3)$ & $3(100)$ & $4(80)$ & \multirow{2}{*}{0.037} \\
\hline Female & $47(24.1)$ & $41(25.6)$ & $2(11.1)$ & $1(16.7)$ & $2(66.7)$ & - & $1(20)$ & \\
\hline \multicolumn{9}{|l|}{ BMI } \\
\hline Mean \pm SD & $21.5 \pm 2.8$ & $21.4 \pm 2.6$ & $22 \pm 3.1$ & $19.6 \pm 3.5$ & $23.5 \pm 4.9$ & $20.3 \pm 1.2$ & $23.5 \pm 3.3$ & - \\
\hline \multicolumn{9}{|l|}{ CTP Score } \\
\hline $\mathrm{A}$ & $116(59.5)$ & 95 (59.4) & $13(72.2)$ & $4(66.7)$ & $2(66.7)$ & $1(33.3)$ & - & \multirow{4}{*}{$\leq 0.001$} \\
\hline B & $62(31.8)$ & $53(33.1)$ & $5(27.8)$ & $2(33.3)$ & $1(33.3)$ & $1(33.3)$ & - & \\
\hline $\mathrm{C}$ & $11(5.6)$ & $10(6.3)$ & - & - & - & $1(33.3)$ & - & \\
\hline No cirrhosis & $6(3.1)$ & $1(0.6)$ & - & - & - & - & $5(100)$ & \\
\hline \multicolumn{9}{|l|}{ BCLC Staging } \\
\hline 0 & $2(1)$ & $2(1.3)$ & - & - & - & - & - & \multirow{5}{*}{0.124} \\
\hline A & $25(12.8)$ & $21(13.1)$ & $2(11.1)$ & $1(16.7)$ & - & - & $1(20)$ & \\
\hline B & 85 (43.6) & 69 (43.1) & 7 (38.9) & $4(66.7)$ & $2(66.7)$ & $1(33.3)$ & $2(40)$ & \\
\hline $\mathrm{C}$ & 65 (33.3) & $55(34.3)$ & $8(44.4)$ & - & $1(33.3)$ & $1(33.3)$ & - & \\
\hline $\mathrm{D}$ & $18(9.2)$ & $13(8.1)$ & $1(5.6)$ & $1(16.7)$ & - & $1(33.3)$ & $2(40)$ & \\
\hline \multicolumn{9}{|c|}{ History of Viral Treatment } \\
\hline $\mathrm{Ye}$ & $51(26.2)$ & $115(71.9)$ & $4(22.2)$ & $2(33.3)$ & - & - & - & \multirow{2}{*}{0.001} \\
\hline No & $144(73.8)$ & $45(28.1)$ & $14(77.8)$ & $4(66.7)$ & $3(100)$ & $3(100)$ & $5(100)$ & \\
\hline \multicolumn{9}{|l|}{ Viral PCR } \\
\hline Positive & $114(58.5)$ & $100(62.5)$ & $11(61.1)$ & $2(33.3)$ & - & - & - & \multirow{2}{*}{$\leq 0.001$} \\
\hline Negative & $81(41.5)$ & $60(37.5)$ & $7(38.9)$ & $4(66.7)$ & $3(100)$ & $3(100)$ & $5(100)$ & \\
\hline
\end{tabular}

spread, antiviral treatment and treatment modalities were also recorded. Treatment strategies were classified into surgical resection, local ablation; RFA and PEI and Transarterial chemoembolization (TACE).
One hundred and sixteen (59.5\%) had compensated cirrhosis, $73(37.4 \%)$ presented with decompensated cirrhosis and only $6(3.1 \%)$ had no cirrhosis. HCV accounted for the bulk of cirrhosis i.e. 160 
(82.1\%) followed by HBV 18 (9.2\%), HVB and HCV coinfection $6(3.1 \%), \mathrm{NASH}$ and cryptogenic cirrhosis 3 (1.5\% each).

Majority of patients 85 (43.6\%) belonged to BCLC B followed by BCLC C 65 (33.3\%), BCLC A 25 (12.8\%) and BCLC D $18(9.2 \%)$ and BCLC 02 (1\%). One hund- red and sixteen $(59.5 \%)$ patients were in CTP class $\mathrm{A}$, $62(31.8 \%)$ in CTP class B and $11(5.6 \%)$ in CTP class C. Child turcotte pugh score were significantly associated with HCC risk factors.

Only $7(3.6 \%)$ patients needed liver biopsy confirmation. Thirty two $(16.3 \%)$ patients had tumor throm-

Table-II: Characteristics of HCC in relation to the cause of cirrhosis.

\begin{tabular}{|c|c|c|c|c|c|c|c|c|}
\hline Variable & Total & $\mathrm{HCV}$ & HBV & HCV/HBV & NASH & Cryptogenic & No cirrhosis & $p$-value \\
\hline \multicolumn{9}{|c|}{ Number of Lesion(s) } \\
\hline 1 & $87(44.6)$ & $69(43.1)$ & $7(38.9)$ & $4(66.7)$ & $2(66.7)$ & $1(33.3)$ & $4(80)$ & \multirow{4}{*}{0.51} \\
\hline 2 & $36(18.5)$ & $32(20)$ & $3(16.7)$ & - & - & - & $1(20)$ & \\
\hline 3 & $8(4.1)$ & $7(4.4)$ & $1(5.6)$ & - & - & - & - & \\
\hline Multiple bilateral & $64(32.8)$ & $52(32.5)$ & $7(38.9)$ & $2(33.3)$ & $1(33.3)$ & $2(66.7)$ & - & \\
\hline \multicolumn{9}{|c|}{ Size of the Largest Lesion (cm) } \\
\hline Mean \pm SD & $5.5 \pm 3.5$ & $5.5 \pm 3.7$ & $6.1 \pm 2.9$ & $5.3 \pm 1.9$ & $4.1 \pm 0.8$ & $9.1 \pm 1.9$ & $7.5 \pm 1.9$ & 0.139 \\
\hline \multicolumn{9}{|c|}{ Portal Vein Thrombosis } \\
\hline Nil & $153(78.5)$ & $121(75.6)$ & $16(88.9)$ & $6(100)$ & $3(100)$ & $2(66.7)$ & $5(100)$ & \multirow{3}{*}{0.46} \\
\hline Bland & $10(5.1)$ & $10(6.3)$ & - & - & - & - & - & \\
\hline Tumor & $32(16.4)$ & $29(18.1)$ & $2(11.1)$ & - & - & $1(33.3)$ & - & \\
\hline \multicolumn{9}{|c|}{ Extra-Hepatic Metastasis } \\
\hline Lymph nodes & $4(40)$ & $2(20)$ & $1(10)$ & - & $1(10)$ & - & - & \multirow{5}{*}{0.55} \\
\hline Adrenals & $2(20)$ & $2(20)$ & - & - & - & - & - & \\
\hline Lungs & $2(20)$ & $2(20)$ & - & - & - & - & - & \\
\hline Spleen and bones & $1(10)$ & $1(10)$ & - & - & - & - & - & \\
\hline Lungs and bones & $1(10)$ & $1(10)$ & - & - & - & - & - & \\
\hline
\end{tabular}

Table-III: Relation of viral PCR and tumor aggressiveness.

\begin{tabular}{|c|c|c|c|}
\hline Variable & $\begin{array}{c}\text { Viral PCR } \\
\text { Positive } \\
\text { (114) }\end{array}$ & $\begin{array}{l}\text { Viral PCR } \\
\text { Negative } \\
\text { (81) }\end{array}$ & $\begin{array}{c}p- \\
\text { value }\end{array}$ \\
\hline \multicolumn{4}{|l|}{ BCLC } \\
\hline 0 & - & $2(2.4)$ & \multirow{5}{*}{0.019} \\
\hline A & $15(13.1)$ & $10(12.3)$ & \\
\hline B & $49(42.9)$ & $36(44.4)$ & \\
\hline $\mathrm{C}$ & $42(36.8)$ & $23(28.3)$ & \\
\hline $\mathrm{D}$ & $8(7.01)$ & $10(12.3)$ & \\
\hline \multicolumn{4}{|l|}{ Tumor Size (cm) } \\
\hline $1-5$ & $57(50)$ & $54(66.6)$ & \multirow{4}{*}{0.19} \\
\hline $6-10$ & $46(40.3)$ & $24(29.6)$ & \\
\hline $11-15$ & $10(8.7)$ & $4(4.9)$ & \\
\hline $16-20$ & $1(0.8)$ & - & \\
\hline \multicolumn{4}{|l|}{ Number of Lesion(s) } \\
\hline 1 & $38(33.3)$ & $49(60.4)$ & \multirow{4}{*}{$\leq 0.001$} \\
\hline 2 & $24(21.0)$ & $12(14.8)$ & \\
\hline 3 & $7(6.1)$ & $1(1.2)$ & \\
\hline Multiple bilateral & $45(39.4)$ & $19(23.4)$ & \\
\hline \multicolumn{4}{|c|}{ Portal Vein Thrombosis } \\
\hline Bland thrombus & $4(3.5)$ & $7(8.6)$ & \multirow{3}{*}{0.036} \\
\hline Tumor thrombus & $20(17.5)$ & $11(13.5)$ & \\
\hline No thrombus & $90(78.9)$ & $63(77.7)$ & \\
\hline $\begin{array}{l}\text { Extra-Hepatic } \\
\text { Metastasis }(n=10)\end{array}$ & $2(20)$ & $8(80)$ & 0.684 \\
\hline
\end{tabular}

Table-IV: Relation of prior antiviral therapy and tumor aggressiveness.

\begin{tabular}{|c|c|c|c|}
\hline Variable & $\begin{array}{c}\text { Antiviral } \\
\text { therapy } \\
\text { given } \\
(n=51)\end{array}$ & $\begin{array}{c}\text { Antiviral } \\
\text { therapy } \\
\text { not given } \\
(\mathrm{n}=144)\end{array}$ & $\begin{array}{c}p- \\
\text { value }\end{array}$ \\
\hline \multicolumn{4}{|l|}{ BCLC } \\
\hline 0 & $2(3.9)$ & - & \multirow{5}{*}{0.02} \\
\hline A & $10(19.6)$ & $14(9.7)$ & \\
\hline B & $20(39.2)$ & $66(45.8)$ & \\
\hline $\mathrm{C}$ & $16(31.3)$ & 49 (34.02) & \\
\hline $\mathrm{D}$ & $3(5.8)$ & $15(10.4)$ & \\
\hline \multicolumn{4}{|l|}{ Tumor Size (cm) } \\
\hline $1-5$ & $33(64.7)$ & $88(61.1)$ & \multirow{4}{*}{0.78} \\
\hline $6-10$ & $16(31.3)$ & $45(31.25)$ & \\
\hline $11-15$ & $1(1.9)$ & $11(7.63)$ & \\
\hline $16-20$ & $1(1.9)$ & - & \\
\hline \multicolumn{4}{|c|}{ Number of Lesion(s) } \\
\hline 1 & $28(54.9)$ & $59(40.9)$ & \multirow{4}{*}{0.135} \\
\hline 2 & $7(13.7)$ & $29(20.1)$ & \\
\hline 3 & $2(3.9)$ & $6(4.1)$ & \\
\hline Multiple bilateral & $14(27.4)$ & $50(34.7)$ & \\
\hline \multicolumn{4}{|c|}{ Portal Vein Thrombosis } \\
\hline Bland thrombus & $2(3.9)$ & $8(5.5)$ & \multirow{3}{*}{0.039} \\
\hline Tumor thrombus & $11(21.5)$ & $21(14.5)$ & \\
\hline No thrombus & $38(74.5)$ & $115(79.8)$ & \\
\hline $\begin{array}{l}\text { Extra-Hepatic } \\
\text { Metastasis }(n=10)\end{array}$ & $1(10)$ & $9(90)$ & 0.989 \\
\hline
\end{tabular}

BCLC Barcelona Clinic of Liver Cancer, $p$ is considered significant if $\leq 0.05$ 
bus in portal vein and 10 (5.1\%) had bland thrombus. Viral PCR was positive for 114 (58.5\%) patients and $132(68 \%)$ patients were treatment naïve. Eighty seven $(44.6 \%)$ had a single lesion, $36(18.5 \%)$ had two lesions whereas multiple bilateral lesions were present in 64 $(32.8 \%)$ patients. The mean \pm SD size of lesion was 5.6 \pm 3.5. Viral PCR status and history of antiviral treatment were significantly associated with risk factors of HCC while number of lesions and size of lesion didn't show any association with HCC etiology or risk factors (table-I).

Relation of viral PCR and prior antiviral treatment with tumor aggressiveness in terms of BCLC classification, number of lesions and portal vein thrombosis showed statistically significant association.

Discuss In this study we have looked into different causes of hepatocellular carcinoma its clinical features, treatment methods, and overall biochemical parameters and behaviour of disease in Pakistan. In accordance with different studies all over the country, HCC was found to be common in fifth and sixth decades and predominantly in males. Chronic HCV infection was most commonly found as cause of HCC after leading to cirrhosis. Hence median age at diagnosis was $60 \pm 9$. It proposes that age difference between $\mathrm{HBV}$ and HCV related hepatocellular carcinoma diagnosis was due to early $\mathrm{HBV}$ infection in peri-natal period, whereas, HCV is acquired in adulthood 8 .

Mostly patients presented in child turcotte pugh class $B$ and in BCLC class C hence revealing the fact that patients were already undergoing severe hepatic dysfunction and poor performance status. CTP class was significantly associated with risk factors for HCC. A significant relationship between viral load and and increased HCC risk and aggressiveness in terms of BCLC classification was seen. Persistent viral replication lead to chronic hepatic inflammation and fibrosis by production of carcinogenic growth factors ${ }^{8}$.

NAFLD is considered the emerging cause of HCC and majority of the cryptogenic cirrhosis is thought to be due to underlying NAFLD 9 . Among non $B$ and non $C$ cases of HCC NAFLD is the leading cause of $\mathrm{HCC}^{10}$. AASLD guidelines recommend that a mass found incidentally or on screening in CLD patients is likely to be $\mathrm{HCC}^{11}$. Hence diagnosis was confirmed by triphasic contrast enhanced CT scan of abdomen in most patients while $3.6 \%$ of patients needed liver biopsy as confirmation because of being not recognizable by imaging modalities in accordance with ASSLD guidelines.
At risk population includes those having cirrhosis. However few non-cirrhotic HCC does occur with viral liver disease especially HBV. Most of the patients in our study were PCR positive $(58.5 \%)$ and $68 \%$ were treatment naïve. The HCC was attributed to $\mathrm{HBV} /$ $\mathrm{HCV}$ coinfection in $3.1 \%$ which is comparable to other national studies ${ }^{12}$.

This shows lack of surveillance and poor trust in health care facilities among masses. This reflects atrisk individuals not being identified, the absence of comprehensive surveillance programs for hepatocellular carcinoma, poor access to expert medical care, an absence of trust in health-care systems and poor health-seeking behavior ${ }^{13}$. Patients with undetectable viral loads following treatment have lower incidence of HCC hence indirectly supporting the association of viral treatment and hepatocellular carcinoma incidence $^{14}$.

Most of the patients at time of presentation had single lesions with size of $5.6 \pm 3.5$. Only $16.3 \%$ had Portal vein thrombosis. Approximately $10-40 \%$ patients with HCC have PVT at the time of diagnosis, and approximately $35-44 \%$ will be found to have PVT at the time of death or liver transplant ${ }^{15}$.

The relative risk of developing PVT in the presence of cirrhosis is almost seven-fold increased above the risk observed in the general population, which is estimated to be $<1.0 \% 15$. Patients with compensated cirrhosis are rarely affected ${ }^{16}$. Several treatment modalities are available namely liver resection, liver transplantation, percutaneous local ablation therapy, and transarterial chemoembolization (TACE). Some of the patients were candidates of systemic therapy namely sorafenib. Tyrosine kinase inhibitors have been approved for the treatment of HCC, sorafenib and second line is regorafenib ${ }^{17}$ but not available in our country.

Few evidence-based guidelines for decision making have been reported throughout the world. Our Patients were selected for appropriate therapies suitable to their co-morbids, life expectancy and in accordance with BCLC criteria. Liver transplantation and Hepatic resection remain as the corner stones for curative therapy of Liver Cancer. However, the success rate of these therapies is for five years ${ }^{18}$.

Alpha feto protein, though not of diagnostic value when $<400 \mathrm{ng} / \mathrm{L}$, was found to be of limited value in terms of sensitivity and specificity while imaging techniques in proper hands are more accurate ${ }^{19}$. Approximately $50 \%$ of HCCs secrete $\mathrm{AFP}^{20}$. 
AFP levels are also frequently elevated in chronic active hepatitis C (levels of $200-300 \mathrm{ng} / \mathrm{mL}$ are not uncommon), but they tend to fluctuate and do not progressively increase.

Biannual ultrasound combined with alpha fetoprotein can well in time detect asymptomatic patients. As cirrhosis takes years to develop after initial contacting virus hence antiviral treatment in time can play a role. Screening and timely treatment of $\mathrm{HBV}$ and $\mathrm{HCV}$ infection and screening for HCC in persons with such infections or cirrhosis of any cause can increase the probability of cure ${ }^{21}$.

\section{CONCLUSION}

Cirrhosis patients are more at risk of developing HCC and our study also supports it. Most patients presented with Child class A and B. More than one third of patients have multifocal HCC as shown by our study too. Mostly afflicted had never received treatment neither were into any surveillance program. Early recognition of at risk population, increasing awareness regarding sources of chronic hepatitis and prevention of liver disease by providing antiviral treatment and implementation of surveillance are essential components of attempts to curb the morbidity and mortality from HCC. Tumor aggressiveness in terms of stage, number of lesions along with PVT showed siginificant statistical association with PCR status and anti-viral treatment.

\section{CONFLICT OF INTEREST}

This study has no conflict of interest to be declared by any author.

\section{REFERENCES}

1. Ghouri YA, Mian I. Review of hepatocellular carcinoma: Epidemiology, etiology \& carcinogenesis. J Carcinog 2017; 16(1): 1-5.

2. Bhatti ABH, Dar FS, Waheed A, Shafique K, Sultan F, Shah NH. Hepatocellular carcinoma in Pakistan: National Trends and Global Perspective. Gastroenterol Res Prac 2016; 2016: 5942306.

3. Zobair M, Younossi. The epidemiology of non alcoholic steatohepatitis. Clin Liver Dis 2018; 11(4): 1-6.

4. Pinter M, Trauner M, Peck-Radosavljevic M, Sieghart W. Cancer and liver cirrhosis: implications on prognosisand management. ESMO Open 2016; 1(1): e000042.
5. Lokesh KN, Chaudhuri T. Advanced hepatocellular carcinoma: Ind J Cancer 2017; 54(3): 526-29.

6. Jin-Young. CT and MRI diagnosis and staging of HCC: public: $\mathrm{CT}$ and MR Imaging diagnosis and staging of hepatocellular carcinoma: Part I. development, growth, and spread: key pathologic and imaging aspects. Radiol 2014; 272(3): 635-54.

7. Parkash O, Hamid S. Next big threat for Pakistan Hepatocellular Carcinoma (HCC). J Pak Med Assoc 2016; 66(6): 735-39.

8. Li-Shuai Qu, Guo-Xiong Zhou. Significance of viral status on occurrence of hepatitis B-related hepatocellular carcinoma: World J Gastroenterol 2014; 20(20): 5999-6005.

9. Piñero F, Pages J, Ridruejo E, Colombato L, Silva M. Fatty liver disease, an emerging etiology of HCC in Argentina. World J Hepatol 2018; 10(1): 41-50.

10. Tateishi R, Okanoue T, Fujiwara N. Clinical characteristics, treatment, and prognosis of non- $\mathrm{B}$, non-C hepatocellular carcinoma: a large retrospective multicenter cohort study. J Gastroenterol 2015; 50(3): 350-60.

11. Marrero JA, Kulik LM, Sirlin CB, Zhu AX, Finn RS, et al. Diagnosis, staging, and management of hepatocellular carcinoma: 2018 practice guidance by the american association for the study of liver diseases. Hepatol 2018; 68(2): 1-8.

12. Butt AS. Epidemiology of viral hepatitis and liver diseases in Pakistan. Euroasian J Hepatogastroenterol 2015; 5(1): 43-48.

13. Yang JD, Mohamed RA, Aziz AOA, Shousha HI, Hashem MB, Nabeel MM, et al. Characteristics, management, and outcomes of patients with hepatocellular carcinoma in Africa: a multicountry observational study from the Africa Liver Cancer Consortium. Lancet 2017; 2(2): P103-111.

14. Mei Lu, Jia Li. Hepatitis C treatment failure is associated with increased risk of hepatocellular carcinoma: J Viral Hepatol 2016; 23(9): 718-29.

15. Quirk M, Kim YH, Saab S, Lee EW. Management of hepatocellular carcinoma with portal vein thrombosis. World J Gastroenterol 2015; 21(12): 3462-71.

16. Köckritz LV, Gottardi AD, Trebicka J, Praktiknjo M. Portal vein thrombosis in patients with cirrhosis. Rep Gastroenterol 2017; 5(2): 148-56.

17. Pinter M, Peck-Radosavljevic M. Review article: systemic treatment of hepatocellular carcinoma: Aliment Pharmacol Ther 2018; 48(6): 598-609.

18. Deepika M. Estimating the risk of hepatocellular carcinoma in developing countries. Res Rev J Med Health Sci 2015; 4(1): 1-8.

19. Forner A, Reig M, Bruix J. Alpha fetoprotein for hepatocellular carcinoma diagnosis. Medicine (Baltimore) 2015; 94(23): 1-5.

20. Bai D, Zhang C, Chen P, Jin S, Jiang G. The prognostic correlation of AFP level at diagnosis with pathological grade, progression, and survival of patients with hepatocellular carcinoma. Sci Rep 2017; 7(1): 12870.

21. Moore MS, Ivanina E, Bornschlegel K, Qiao B, Schymura MJ, Laraque F. Hepatocellular carcinoma and viral hepatitis in New York City. Clin Infect Dis 2016; 63(12): 1577-83. 